ESTUDIOS / RESEARCH STUDIES

\title{
Rankings nacionales elaborados a partir de múltiples indicadores frente a los de índices sintéticos
}

\author{
Elías Sanz-Casado*,**, Carlos García-Zorita*,**, Antonio Eleazar Serrano-López* , \\ Preiddy Efraín-García*, Daniela De Filippo* \\ *Laboratorio de Estudios Métricos de la Información (LEMI), Departamento de Biblioteconomía y Documentación. \\ Universidad Carlos III de Madrid, España. \\ ** Unidad Asociada IEDCYT-LEMI. CSIC-Universidad Carlos III de Madrid \\ Correo-e: elias@bib.uc3m.es
}

Recibido: 20-09-2012; 2a versión: 18-02-2013; Aceptado: 19-02-2013

Cómo citar este artículo/ Citation: Sanz-Casado, E.; García-Zorita, C.; Serrano-López, A. E.; Efraín-García, P.; De Filipo, D. (2013). Rankings nacionales elaborados a partir de múltiples indicadores frente a los de índices sintéticos. Revista Española de Documentación Científica, 36(3):e012. doi: http://dx.doi.org/10.3989/redc.2013.3.1.023

Resumen: En este trabajo se analiza el interés de los rankings nacionales de universidades para visibilizar la actividad investigadora de estas instituciones en países no centrales. Para ello, en la primera parte de carácter metodológico, se presentan las características de los rankings nacionales que se muestran más adecuados para identificar y comparar las actividades de las instituciones de un mismo entorno. Se describen, además, las ventajas y limitaciones de la utilización de múltiples indicadores frente a indicadores sintéticos. En la segunda parte se presenta el Observatorio IUNE como una herramienta que ofrece información amplia y variada para el seguimiento de la actividad investigadora de las universidades españolas. Los datos obtenidos permiten conocer las características del sistema universitario español, así como posicionar a las instituciones en función de su actividad en 6 dimensiones diversas a partir de 42 indicadores distintos.

Palabras clave: Rankings de universidades; actividad científica; Observatorio IUNE; indicadores.

\section{National Rankings based on multiple indicators versus synthetic indices}

Abstract: This paper analyzes the interest of national rankings of universities in making visible the research activities of these institutions in non-core countries. To do this, the first part of methodology presents the characteristics of the national rankings that are more appropriate for identifying and comparing the activities of institutions of the same environment. It also describes the advantages and limitations of using multiple indicators as opposed to synthetic indicators. The second part presents the IUNE Observatory as a tool that provides comprehensive and diverse information for monitoring the research activity of Spanish universities. The data obtained allow us to identify the characteristics of the Spanish university system as well as to position the institutions according to their activities, into 6 different dimensions based on 42 different indicators.

Keywords: Universities rankings; scientific activity; IUNE observatory; indicators.

Copyright: (c) 2013 CSIC. Este es un artículo de acceso abierto distribuido bajo los términos de la licencia Creative Commons Attribution-Non Commercial (by-nc) Spain 3.0. 


\section{INTRODUCCIÓN}

Procesos de evaluación de la actividad científica en las universidades

La relevancia del análisis y evaluación de la actividad investigadora se ha puesto de manifiesto a través de los diferentes procesos llevados a cabo por diversas instituciones. El Reino Unido fue uno de los primeros países en reconocer la complejidad de la actividad investigadora del sistema universitario, así como de la necesidad de evaluarla, y ha venido desarrollando desde 1986 procesos específicos de evaluación adaptados a este ámbito como el Research Assessment Exercise (RAE). Si bien este proceso de evaluación se ha basado fundamentalmente en paneles de expertos, entre las recomendaciones de la convocatoria del año 2001, se propone de manera explícita la utilización en el proceso evaluador de indicadores bibliométricos, con el fin de complementar la información relevante que están utilizando los expertos.

Otra iniciativa similar fue la iniciada en 1999 por la Universidad de Helsinki -que se repite cada 6 años- con el objetivo de conocer y consolidar su posición científica entre las universidades líderes europeas. El proceso de evaluación se realiza, al igual que el RAE, a partir de paneles de expertos y tiene como objetivo consolidar la posición científica de esta Universidad entre las universidades líderes europeas.

También hay que señalar el proceso de evaluación de las universidades australianas, dentro del Research Evaluation Policy Project (REPP). Este proyecto comenzó en 2004, y tuvo por objeto analizar la calidad de la investigación realizada por las instituciones de educación superior de ese país, con el fin de realizar un reparto de los fondos disponibles en función de la calidad. En este caso, además de la evaluación por pares, se utilizaron indicadores métricos (bibliométricos y cienciométricos), que han demostrado ser eficientes en la aportación de gran cantidad de datos al proceso evaluador.

En España, uno de los procesos de evaluación de la actividad investigadora del profesorado de las universidades que se realiza de manera periódica y sistemática, es el llevado a cabo por la Comisión Nacional Evaluadora de la Actividad Investigadora (CNEAI), que evalúa 6 años de actividad de los investigadores que deciden someterse a este proceso de manera voluntaria. Otro proceso de evaluación, aunque en este caso a nivel departamental, ha sido realizado por el Ministerio de Educación dentro del Plan de Calidad de la Universidades. Estas dos evaluaciones se realizan fundamentalmente a partir de expertos, aunque en ellas se tienen en cuenta las publicaciones y las citas.

La evaluación de la actividad científica del sistema universitario español también ha sido abordada, aunque no de manera periódica y sistemática, a partir de otras técnicas como son los análisis bibliométricos y cienciométricos, o la utilización de encuestas dirigidas al profesorado. Trabajos de este tipo han sido realizadas por grupos de investigación del Instituto de Estudios Documentales sobre Ciencia y Tecnología (IEDCYT) (Gómez y otros, 2009), así como por grupos de investigación de otras instituciones como la Universidad de Granada, concretamente los grupos de Evaluación de la Ciencia y de la Comunicación Científica de la Facultad de Comunicación y Documentación (EC3) (Torres-Salinas y Cabezas-Clavijo, 2012; Torres Salinas y otros, 2011a), y el liderado por Buela Casal de la Facultad de Psicología de la citada Universidad (Buela-Casal y otros, 2010, Buela-Casal y otros, 2011). También hay que señalar los trabajos realizados por el grupo de investigación Scimago sobre la actividad investigadora del sistema universitario español (Corera y otros, 2010). Finalmente, otros estudios sobre este sector científico serían los realizados por el Laboratorio de Estudios Métricos de Información (LEMI) de la Universidad Carlos III de Madrid (Sanz-Casado y otros, 2008).

\section{Rankings regionales frente a rankings} internacionales de universidades

En la última década, el rápido desarrollo de los rankings de universidades ha demostrado que pueden ser una herramienta útil, y por tanto cada vez más utilizada a la hora de evaluar la actividad investigadora de este tipo de instituciones.

Si bien el debate sobre la validez, utilidad e importancia de los rankings de universidades es un tema de suma actualidad, el desarrollo de estos rankings o listados de instituciones no es un fenómeno nuevo. Hacia 1910 James McKeen Cattel inició la tradición de los listados de universidades analizando las instituciones de Estados Unidos y utilizando como indicador de la calidad la afiliación institucional de científicos destacados. En 1925 Hughes publicó un listado de universidades basado en opiniones de expertos y, una década más tarde, repitió este ejercicio mejorando la metodología (Webster, 1983; Webster, 1986).

Entre las décadas de los 60 y los 80 los listados de universidades norteamericanas tuvieron un desarrollo continuo y desde los 90 se volvieron parte del bagaje de quienes aspiran a hacer estudios superiores o a elaborar rankings anuales de instituciones o programas en muchos países del mundo (Martínez Rizo, 2012).

Como explica Webster, uno de los antecedentes de los rankings internacionales de universidades puede encontrarse en los listados publicados por Jack Gourman entre 1967 y 1983, en los que pretendía listar en función de la calidad a un centenar de programas de unas 1500 universidades de Estados Unidos y 700 del resto del mundo (Webster, 1985). Sin embargo, es a comienzos del siglo XXI cuando los rankings internacionales adquieren gran relevancia y visibilidad, en parte gracias a su enorme difusión vía Internet. Una de 
las iniciativas de mayor impacto ha sido la llevada a cabo por la Jiao Tong University en Shanghái que en 2003 lanzó el Academic Ranking of World Universities (ARWU) - más conocido como ranking de Shanghái- con el fin de evaluar la actividad científica de las universidades y construir un ranking en función de su calidad. Desde entonces, muchas han sido las propuestas surgidas en todo el mundo. Entre ellas el Leiden Ranking, QS World University Ranking, Times Higher Education Ranking, CHE Ranking, Scimago Institutions Rankings, y el Ranking Web of World Universities (Torres-Salinas y otros, 2011a). A estos rankings hay que añadir dos más en el ámbito español el Ranking I-UGR de Universidades Españolas del que es responsable el grupo EC3 de la Universidad de Granada (http:// www.rankinguniversidades.es), y el Observatorio de la Actividad Investigadora de la Universidad Española (IUNE) elaborado por la Alianza de las 4 Universidades (http://www.iune.es).

Según los datos ofrecidos en el International Handbook of Universities (2012), actualmente existen en el mundo alrededor de 15.000 universidades. Si bien el gran número de instituciones de educación superior ha sido un factor importante para el desarrollo y la creciente popularidad de los rankings (Egron-Polak, 2007), algunos aspectos como la diversidad de sus funciones y programas, su naturaleza pública o privada y las propias diferencias culturales o sociales entre regiones, hacen que la definición misma de "universidad" y la confección de un catálogo mundial exhaustivo y riguroso resulte una tarea compleja (Orduña, 2011).

Asimismo, aunque muchos de estos rankings han logrado reconocimiento internacional y son tenidos en cuenta para la toma de decisiones, su creación y uso no están exentos de controversias. Por una parte su metodología ha sido ampliamente debatida ya que la selección de indicadores, su ponderación, tratamiento de los datos y la transparencia en la obtención de resultados evidencian grandes limitaciones (Liu y Cheng, 2005; van-Raan, 2005; Buesa y otros 2009).

Además de las dificultades metodológicas, otra limitación importante es que en los rankings internacionales solo aparece un número reducido de instituciones de países no centrales. Esto sucede porque muchos rankings toman como modelo de universidad las de Estados Unidos (o de los países anglosajones) en los que predomina un tipo de universidad más elitista y orientada fundamentalmente a la investigación. Este modelo impide detectar la diversidad de tipologías que existen en otros contextos y que resultan igualmente valiosas para el desarrollo integral de su entorno. En ese sentido, como comenta Muñoz García (2012), resulta muy relevante tener en cuenta la diversidad de las situaciones nacionales, ya que las universidades y los sistemas de educación superior se desarrollan de cara a las necesidades y prioridades de los países, según las legislaciones que ordenan su organización y funcionamiento y de acuerdo con políticas de Estado propias de quienes controlan el gobierno y el poder público. "Así a las instituciones universitarias hay que entenderlas como parte de sistemas nacionales de educación superior. El análisis comparativo de la educación superior demanda reconocer que hay diferencias entre países en los sistemas educativos y en las universidades" (Clark, 1997; Marginson y Mollis, 2001).

Otra dificultad añadida es la obtención de determinados indicadores que en algunos rankings juegan un papel importante en la posición final de las instituciones y que solo afectan a unas pocas universidades del mundo, como son los premios Nobel o medallas Fields concedidos a los estudiantes o al profesorado.También el escaso peso que se da al tamaño de las instituciones perjudica aquellas pequeñas o medianas que aunque sean muy eficientes en su actividad investigadora, les resulta muy difícil competir con aquellas otras de gran tamaño que con menor esfuerzo tienen mucha mayor facilidad de colocarse en las posiciones más avanzadas. Todo ello contribuye a que de forma prácticamente constante año tras años se repita una concentración importante de universidades norteamericanas y de unos pocos países europeos y asiáticos en las primeras posiciones de algunos de los rankings internacionales de mayor prestigio.

Es por ello que complementar los datos obtenidos en los rankings internacionales con iniciativas nacionales puede ser una buena estrategia a la hora de analizar la posición de una institución tanto en el contexto internacional como dentro de su propio entorno.

Con la intención de realizar comparaciones entre instituciones similares en ámbitos nacionales han surgido distintas iniciativas; por ejemplo, en España se han elaborado diversos rankings como el Índice multidimensional de la calidad de las universidades (Buesa y otros, 2009), el Ranking de investigación de las universidades públicas españolas (Buela-Casal y otros, 2011), el Ranking General y por áreas de las instituciones universitarias españolas (Corera y otros, 2010), el Ranking I-UGR de Universidades Españolas (Torres-Salinas y otros, 2011a), o el Observatorio de la Actividad Investigadora de la Universidad Española (IUNE).

A pesar de los importantes avances que supone para el contexto local contar con esta información, algunas limitaciones continúan presentes. Entre ellas, el hecho de que en algunos rankings sólo se analicen determinados campos científicos, se incluya un período temporal reducido, se consideren sólo unos pocos indicadores, o se estudie solo un grupo de instituciones del sistema universitario. La selección arbitraria de fuentes o la elaboración de índices sintéticos hacen que los resultados no resulten completamente óptimos. A su vez, al estudiar la actividad investigadora de las universidades, en muchos casos, se utilizan únicamente indicadores bibliométricos o asociados a la producción 
de publicaciones científicas (número de publicaciones, citaciones, artículos en revistas top, etc). Esto ofrece una visión muy fragmentaria al mostrar sólo una única dimensión de la actividad investigadora de las universidades.

Rankings de múltiples Indicadores versus rankings de índices sintéticos

Cada vez con mayor frecuencia los gestores y evaluadores recurren a los rankings de universidades, ya que permiten conocer de manera rápida y sencilla cuestiones vinculadas con la actividad y el rendimiento de las diferentes instituciones, permitiéndoles determinar algunos puntos fuertes y débiles de esta actividad científica, así como realizar estudios de comparabilidad de resultados entre las instituciones. En este sentido, la creación de rankings en los que se representa un conjunto de variables a partir de indicadores unidimensionales ha resultado muy útil. Sin embargo, no hay que perder de vista que esto también conlleva ciertas limitaciones.

Tal como comenta Marginson (2012) todas las ponderaciones que se realicen para otorgar pesos a las diferentes variables y obtener un índice único, son elaboraciones subjetivas y arbitrarias producidas a partir de la importancia que diversos actores dan a priori a estas diferentes dimensiones $y$, por lo tanto, al reducir variables, ocultan la diversidad propia de las distintas instituciones.

En el caso de las actividades de I+D desarrolladas en el sistema universitario, por ejemplo, existe un conjunto muy variado de actividades a evaluar y que se encuentran íntimamente relacionadas con las características estructurales de cada universidad, como el tamaño o el tipo de institución (públi$\mathrm{ca} /$ privada). A su vez, la especialización temática de cada institución puede favorecer la orientación hacia la realización de determinadas actividades muy relacionadas con su campo científico, por lo que en los análisis globales y teniendo en cuenta índices sintéticos las posiciones de estas universidades se ven perjudicadas por este hecho. En este sentido y en un contexto en el que la utilización de la información aportada por los rankings de universidades es cada vez más utilizada como herramienta de evaluación institucional, resulta fundamental recordar sus limitaciones para no caer en prácticas reduccionistas.

A nivel europeo han surgido en los últimos años propuestas como el proyecto U-Map que ha realizado una clasificación de instituciones de educación superior según diferentes dimensiones como: perfil educativo; perfil de los estudiantes; actividades de investigación; transferencia de conocimientos; internacionalización y relación con el entorno local. A través de estas dimensiones no se intenta obtener un ranking institucional, sino ofrecer una batería de indicadores para posicionar a cada institución en función de su propio perfil (van Vught y otros, 2010).

En esta misma línea se ha desarrollado el proyecto U-Multirank que ya ha completado el estudio piloto en 3 disciplinas en 150 instituciones de 50 países para realizar comparaciones en las dimensiones de actividad identificadas previamente en U-Map. Con esta iniciativa se supera también la noción de rankings y se ofrece una selección de múltiples indicadores haciendo mucho más flexible y variado el estudio de las diversas instituciones (van Vught y Ziegele, 2011).

Asimismo, en el ámbito Latinoamericano se han venido desarrollando diferentes iniciativas para generar información que permita analizar y evaluar a las universidades de la región desde una perspectiva mucho más adecuada a su realidad. Una de ellas es la puesta en marcha del Mapa de la Educación Superior en América Latina y el Caribe (MESALC) en el marco de las actividades desarrolladas por el Instituto Internacional para la Educación Superior en América Latina y el Caribe (IESALC) de la UNESCO. Esta iniciativa ha surgido a partir de la detección de la escasa visibilidad de las universidades de la región en los rankings internacionales debido a la utilización de criterios para su elaboración muy alejados de la realidad de estas instituciones, pero que, sin embargo, tienen un impacto muy marcado en la toma de decisiones.

Teniendo en cuenta el impacto y la utilidad que tienen los rankings de universidades en el nuevo contexto en el que se tienen que desenvolver este tipo de instituciones, pero considerando también sus debilidades, en España se ha desarrollado el Observatorio de la Actividad Investigadora en la Universidad Española (IUNE), con la intención de superar muchas de las limitaciones planteadas. El Observatorio IUNE se ha creado con el fin de realizar el análisis y seguimiento de la actividad investigadora de las universidades españolas desde distintas perspectivas, presentando algunos aspectos metodológicos que lo diferencia de otros rankings. En la tabla I se muestran algunas de estas limitaciones y las soluciones aportadas por el Observatorio IUNE.

A partir de los comentarios realizados hasta el momento y teniendo en cuenta la relevancia de la discusión sobre la validez de los rankings de universidades, los objetivos que se pretenden alcanzar con este trabajo son los siguientes:

1- Analizar la actividad investigadora de las universidades públicas españolas, a partir de un conjunto de indicadores relativos a las dimensiones más significativas vinculadas con dicha actividad.

2- Mostrar el interés del Observatorio IUNE como fuente de obtención de datos para caracterizar la actividad científica del sistema universitario español, a partir de la utilidad y exhaustividad de los indicadores que incluye.

\section{FUENTES Y METODOLOGÍA}

Se ha utilizado como fuente de información el Observatorio IUNE. A través del portal web del Ob- 
Tabla I. Fortalezas del Observatorio IUNE frente a limitaciones de otros rankings

\begin{tabular}{|c|c|}
\hline Limitaciones & Observatorio IUNE \\
\hline Selección arbitraria de fuentes de información & $\begin{array}{l}\text { Uso exclusivo de fuentes de información contrastadas, fiables y } \\
\text { accesibles }\end{array}$ \\
\hline Falta de actualización de los datos & Actualización 2 veces al año \\
\hline Período de análisis reducido & Datos desde el año 2002 (actualmente hasta 2010) \\
\hline Definición poco clara de las instituciones incluidas & $\begin{array}{l}\text { Análisis de todo el sistema universitario español (público y } \\
\text { privado) }\end{array}$ \\
\hline $\begin{array}{l}\text { Indicadores relacionados con una (o pocas) } \\
\text { dimensiones de la actividad científica }\end{array}$ & Inclusión de 6 dimensiones y 42 indicadores \\
\hline $\begin{array}{l}\text { Dudoso reconocimiento de la institución que lo elabora } \\
\text { (en ocasiones fundaciones privadas, consultoras) }\end{array}$ & $\begin{array}{l}\text { Elaboración por parte de un equipo de investigación } \\
\text { interdisciplinar de reconocido prestigio y avalado por el Ministerio } \\
\text { de Educación }\end{array}$ \\
\hline $\begin{array}{l}\text { Complejidad y falta de transparencia en el cálculo de } \\
\text { indicadores }\end{array}$ & $\begin{array}{l}\text { Definición de indicadores claros, sencillos y comparables. } \\
\text { Discusión y contraste de los indicadores seleccionados con } \\
\text { expertos. }\end{array}$ \\
\hline Análisis de unos pocos campos científicos & $\begin{array}{l}\text { Información sobre la actividad de las universidades en todas las } \\
\text { áreas del conocimiento }\end{array}$ \\
\hline $\begin{array}{l}\text { Fallos en el tratamiento de los datos que dificultan } \\
\text { la asignación de resultados a las instituciones } \\
\text { correspondientes }\end{array}$ & $\begin{array}{l}\text { Depuración, homologación y revisión de datos con técnicas } \\
\text { específicas para una asignación precisa a las instituciones } \\
\text { correspondientes }\end{array}$ \\
\hline Presentación estática de resultados & $\begin{array}{l}\text { Creación de una plataforma web dinámica que permite visualizar } \\
\text { datos anuales, de un período y obtener gráficos y tablas de } \\
\text { manera automática }\end{array}$ \\
\hline
\end{tabular}

servatorio (www.iune.es) se ha accedido a los indicadores incluidos en cada dimensión, obteniéndose un listado con la posición en la que se ubica cada universidad en las diferentes variables analizadas. Esta fuente ofrece información sobre 48 universidades públicas y 25 privadas durante el período 2002-2010, aunque en este trabajo solo se han considerado las públicas.

Se ha realizado una selección de indicadores de cada una de las 6 dimensiones incluidas en IUNE con el fin de mostrar los principales resultados de la actividad investigadora de las universidades públicas españolas durante el período 2002-2010. En el Anexo I se presenta un listado de acrónimos de las universidades públicas.

Las dimensiones e indicadores analizados son los siguientes:

- Profesorado: número de profesores permanentes de las universidades públicas

- Reconocimiento: número de sexenios obtenidos por universidad

- Actividad investigadora: productividad, colaboración internacional, impacto y visibilidad

- Innovación: número de patentes solicitadas

Competitividad: número de proyectos del Plan Nacional y del Programa Marco de la Unión Europea

- Capacidad formativa para la investigación: tesis defendidas
En la tabla II se muestran cada una de estas dimensiones, los indicadores obtenidos y las fuentes de las que se obtienen los datos.

Los datos de profesorado, reconocimiento, innovación, competitividad y capacidad formativa, se obtuvieron seleccionando a través de la descarga directa de datos los nombres de cada universidad. En el caso de los datos relacionados con actividad científica fue necesario llevar a cabo un procedimiento más complejo: En primer lugar se descargaron todos los documentos que contenían "Spain" en el campo "address" de las bases de datos WoS. Mediante un sistema de normalización desarrollado en el Laboratorio de Estudios Métricos de la Información (LEMI) de la Universidad Carlos III de Madrid, se identificaron todas las direcciones únicas (cadenas de texto separadas por punto y coma en las que se consignan las afiliaciones institucionales de los autores) de cada documento. Si en la dirección única aparecía más de una institución se optó por hacer una asignación estricta, de modo que el registro quedará adscrito a una universidad solo en el caso de que en dicha dirección única no se consignara además una institución hospitalaria o un centro mixto del CSIC.

A diferencia de otras metodologías de normalización basadas en master list de variantes de direcciones únicas o en asignaciones a cadena truncadas que toman solo en consideración el texto de la dirección hasta el primer separador (habitualmente una coma), en nuestro caso, se emplean scripts programados en lenguaje Perl que actúan sobre el 
Tabla II. Dimensiones, indicadores y fuentes de información utilizadas en el Observatorio IUNE

\begin{tabular}{|c|c|c|}
\hline Dimensión & Indicador & Fuente de los datos \\
\hline Profesorado & Número de profesores permanentes & INE (Instituto Nacional de Estadística) \\
\hline Reconocimiento & $\begin{array}{c}\text { Número de sexenios obtenidos; Premios Nacionales de } \\
\text { Investigación }\end{array}$ & $\begin{array}{c}\text { CNEAI (Comisión Nacional Evaluadora } \\
\text { de la Actividad Investigadora) } \\
\text { ME (Ministerio de Educación, Cultura y } \\
\text { Deporte) }\end{array}$ \\
\hline $\begin{array}{l}\text { Actividad } \\
\text { científica }\end{array}$ & $\begin{array}{l}\text { Producción y productividad, Colaboración, Impacto de la } \\
\text { investigación, Visibilidad }\end{array}$ & $\begin{array}{c}\text { Plataforma Web of Science (Science } \\
\text { Citation Index, Social Sciences Citation } \\
\text { Index, y Arts \& Humanities Citation } \\
\text { Index) }\end{array}$ \\
\hline Innovación & $\begin{array}{l}\text { Número de patentes nacionales; Ingresos generados por } \\
\text { licencias; Número de extensiones PCT; Número de spin-off }\end{array}$ & $\begin{array}{l}\text { INVENES (creada por la Oficina } \\
\text { Española de Patentes y Marcas) } \\
\text { Encuesta anual de la Red OTRI }\end{array}$ \\
\hline Competitividad & $\begin{array}{l}\text { Número de proyectos del Plan Nacional; proyectos } \\
\text { obtenidos de los Programas Marco de UE; }\end{array}$ & $\begin{array}{l}\text { CDTI (Centro para el Desarrollo } \\
\text { Tecnológico Industrial) }\end{array}$ \\
\hline $\begin{array}{l}\text { Capacidad } \\
\text { formativa para la } \\
\text { investigación }\end{array}$ & $\begin{array}{l}\text { Número de Becas FPI; FPU; contratos Juan de la Cierva; } \\
\text { contratos Ramón y Cajal; Tesis defendidas }\end{array}$ & $\begin{array}{c}\text { INE (Instituto Nacional de Estadística) } \\
\text { ME (Ministerio de Educación) } \\
\text { MINECO (Ministerio de Economía y } \\
\text { Competitividad) }\end{array}$ \\
\hline
\end{tabular}

texto completo de dicha dirección. En una primera fase, se utilizan expresiones booleanas para interrogar el registro de dirección empleando términos que identifiquen los diferentes nombres de las universidades españolas. En fases posteriores, para aquellas direcciones no asignadas, se combinan en las búsquedas nombres abreviados y direcciones postales.

\section{RESULTADOS}

En este apartado se presentan los valores de los indicadores de las universidades públicas que se ubican en las 10 primeras posiciones en cada variable de las dimensiones consideradas en el estudio. Las tablas completas con la información de las posiciones de todas las universidades públicas españolas en cada uno de los indicadores analizados se pueden ver pinchando el enlace que parece en el título de cada una de las tablas.

\subsection{Dimensión 1: Profesorado \\ Número de profesores permanentes}

Los datos referentes al profesorado permanente, aquí se han incluido Catedráticos y Titulares de Universidad y de Escuela Universitaria, y Contratados Doctor, muestran que el volumen de personal permanente difiere notablemente de unas universidades a otras. Las que presentan un promedio mayor de profesores en los 9 años analizados son: La Universidad Complutense de Madrid (UCM) con 3.816 profesores, la Universidad de Barcelona (UB) con 2.687, la Universidad Politécnica de Madrid (UPM) con 2.481, la Universidad del País Vasco (EHU) con 2.474, la Universidad de Sevilla (US) con 2.398 profesores y la Universidad de Valencia (UV) con 2.248 (tabla III). Por otro lado, las uni- versidades con un menor promedio de profesores son: La Universidad Pompeu Fabra (UPF) con 285, la Universidad de la Rioja (URIOJA) con 263, y la Universidad Pablo de Olavide (UPO) con 184. Es fundamental considerar esta información, ya que el volumen de profesorado permite caracterizar el tamaño de la universidad, siendo el denominador utilizado en el cálculo de todos los indicadores relativos, con el fin de eliminar los efectos derivados del tamaño de las instituciones.

\subsection{Dimensión 2: Reconocimiento}

\section{Número de sexenios obtenidos}

Los últimos datos disponibles sobre el número de sexenios concedidos fueron publicados en 2010 y corresponden a la actividad del año 2007, pero se refiere a sexenios acumulados, por lo tanto, solo ha sido posible contar con información desagregada por años hasta 2006. En la tabla IV se muestra la evolución de los tramos de investigación concedidos por 100 profesores en cada una de las universidades públicas. Al relativizar los datos al número de profesores, se observa que, en general, las universidades grandes no están entre las primeras posiciones, por lo que el tamaño de la universidad afecta directamente a esta variable. Las universidades con valores superiores a los 12 sexenios por cada 100 profesores son la Pablo de Olavide (UPO) con 16,65 sexenios, la Carlos III de Madrid (UC3M) con 16,25 sexenios, la Miguel Hernández (UMH) con 12,22 y la Autónoma de Madrid (UAM) con 12,01(tabla IV). En el sentido contrario, con menos de 7 sexenios de promedio por cada 100 profesores se encuentra la Universidad de Las Palmas de Gran Canaria (ULPGC) con 6,24 sexenios, la Universidad de Burgos (UBU) con 5,40, y la Universidad Politécnica de Madrid (UPM) con 4,83 
sexenios. Evidentemente, la posición de algunas universidades, sobre todo las politécnicas, está penalizada por la orientación de su actividad científica, más vinculada con la innovación y transferencia a los ámbitos empresariales $y$, por tanto, con una menor actividad publicadora en revistas incluidas en bases de datos con índices de impacto.

\subsection{Dimensión 3: Actividad científica \\ Productividad}

Entre los años 2002 y 2010 la evolución de las publicaciones de las universidades públicas españolas ha experimentado un crecimiento del 91\%, mientras que el incremento de la contratación de profesorado permanente ha sido del $15,74 \%$. Sin embargo, a la hora de analizar la productividad de cada universidad el tamaño de cada una de ellas resulta central. Considerando el número de documentos por profesor durante el período de estudio, se puede observar que entre las más productivas destaca la Universidad Pompeu Fabra (UPF) con 1,47 publicaciones/profesor, seguida de la Univer- sidad Autónoma de Barcelona (UAB) con 1,07, y la Universidad Miguel Hernández (UMH) con 0,88 publicaciones/profesor (tabla $\mathrm{V}$ ). En las últimas posiciones, en cuanto a productividad, se ubican la Universidad Politécnica de Madrid (UPM) con 0,30 publicaciones/profesor, la UNED con 0,29, y la Universidad de las Palmas de Gran Canaria (ULPGC) con 0,27 . En este caso, también la productividad estaría vinculada con la mayor orientación de la universidad hacia determinadas áreas de investigación; por ejemplo las politécnicas, que como se ha comentado, en general presentan menor actividad publicadora, así como con las instituciones con mayor dedicación a las áreas de ciencias sociales y humanidades, cuyas publicaciones están muy mal recogidas en la WoS. Asimismo, existen otros factores que pueden afectar la productividad, como por ejemplo las políticas de contratación de cada universidad. En los casos en los que se opte por mantener una plantilla reducida de profesorado permanente, los valores de rendimiento serán más destacados como en el caso de la UPF.

Tabla III. Número de profesores de las universidades públicas (10 primeras universidades). [Ver tabla completa]

\begin{tabular}{cccccccccccc}
\hline Orden & Universidad & $\mathbf{2 0 0 2}$ & $\mathbf{2 0 0 3}$ & $\mathbf{2 0 0 4}$ & $\mathbf{2 0 0 5}$ & $\mathbf{2 0 0 6}$ & $\mathbf{2 0 0 7}$ & $\mathbf{2 0 0 8}$ & $\mathbf{2 0 0 9}$ & $\mathbf{2 0 1 0}$ & Promedio \\
\hline $\mathbf{1}$ & UCM & 3498 & 3611 & 3665 & 3779 & 3850 & 3988 & 3995 & 4027 & 3931 & 3816 \\
\hline $\mathbf{2}$ & UB & 2531 & 2655 & 2777 & 2768 & 2706 & 2731 & 2704 & 2674 & 2641 & 2687 \\
\hline $\mathbf{3}$ & UPM & 2415 & 2385 & 2446 & 2480 & 2498 & 2470 & 2511 & 2569 & 2554 & 2481 \\
\hline $\mathbf{4}$ & EHU & 2454 & 2591 & 2572 & 2497 & 2467 & 2400 & 2406 & 2355 & 2523 & 2474 \\
\hline $\mathbf{5}$ & US & 2196 & 2211 & 2228 & 2405 & 2437 & 2474 & 2517 & 2559 & 2559 & 2398 \\
\hline $\mathbf{6}$ & UV & 2078 & 2260 & 2253 & 2248 & 2259 & 2296 & 2315 & 2289 & 2234 & 2248 \\
\hline $\mathbf{7}$ & UGR & 2075 & 2114 & 2151 & 2169 & 2146 & 2253 & 2412 & 2444 & 2446 & 2246 \\
\hline $\mathbf{8}$ & UPV & 1628 & 1667 & 1738 & 1737 & 1725 & 1742 & 1809 & 1845 & 1862 & 1750 \\
\hline $\mathbf{9}$ & UNIZAR & 1581 & 1566 & 1728 & 1732 & 1730 & 1749 & 1791 & 1833 & 1812 & 1725 \\
\hline $\mathbf{1 0}$ & USC & 1397 & 1437 & 1518 & 1540 & 1489 & 1590 & 1589 & 1646 & 1695 & 1545 \\
\hline
\end{tabular}

Tabla IV. Sexenios obtenidos por las universidades públicas por 100 profesores (10 primeras universidades). [Ver tabla completa]

\begin{tabular}{|c|c|c|c|c|c|c|c|c|}
\hline Orden & Universidad & 2002 & 2003 & 2004 & 2005 & 2006 & $\begin{array}{c}\text { Promedio / } \\
100 \text { profesores }\end{array}$ & $\begin{array}{c}\text { Total } \\
\text { sexenios }\end{array}$ \\
\hline 1 & UPO & 22 & 25 & 15 & 11 & 11 & 16,8 & 111 \\
\hline 2 & UC3M & 18 & 17 & 14 & 16 & 16 & 16,2 & 460 \\
\hline 3 & UMH & 16 & 10 & 13 & 12 & 10 & 12,2 & 274 \\
\hline 4 & UAM & 17 & 9 & 10 & 9 & 15 & 12 & 1248 \\
\hline 5 & UPF & 16 & 8 & 11 & 11 & 12 & 11,6 & 248 \\
\hline 6 & UJI & 11 & 11 & 17 & 8 & 10 & 11,4 & 358 \\
\hline 7 & UAB & 16 & 11 & 9 & 10 & 10 & 11,2 & 1203 \\
\hline 8 & USC & 10 & 12 & 12 & 7 & 13 & 10,8 & 1187 \\
\hline 9 & UPCT & 17 & 14 & 8 & 5 & 9 & 10,6 & 175 \\
\hline 10 & UV & 13 & 10 & 9 & 9 & 11 & 10,4 & 1744 \\
\hline
\end{tabular}




\section{Colaboración internacional}

Los indicadores de colaboración institucional permiten conocer el perfil de publicación de cada institución a partir de las redes de investigación que establece, tanto en el contexto nacional como internacional. Es, sin embargo, un dato orientativo, dado que la especialización temática de cada universidad afecta considerablemente a sus hábitos de colaboración. En este trabajo se ha decidido analizar solo el perfil internacional de las universidades públicas, presentándose en la tabla VI los resultados relativos a la colaboración internacional de las 10 universidades con mayor actividad. En la página web de IUNE es posible, además, contar con información sobre la colaboración nacional, así como sobre el índice de co-autoría y el grado de colaboración institucional.

El porcentaje de documentos en colaboración internacional es uno de los indicadores más utilizados para analizar la apertura e internacionalización de una institución. En el caso de las universidades públicas españolas, las 5 que muestran un mayor porcentaje de documentos en colaboración con instituciones extranjeras, son las universidades Pompeu Fabra (UPF) con el $51 \%$ de los documentos, la de Barcelona (UB) con el 46\%, la Autónoma de Madrid (UAM) con el 43\%, la Autónoma de Barcelona (UAB) con el $42,70 \%$, y la Politécnica de Cataluña (UPC) con el $42 \%$ (tabla VI). Si tenemos en cuenta que el promedio de colaboración internacional de las universidades públicas es de $33,85 \%$, superarían este umbral 19 de ellas.

\section{Impacto de la investigación}

Aunque no está exento de controversia, el número de citas recibidas es un indicador ampliamente aceptado para conocer el impacto de la producción científica publicada, y de algún modo esta medida se ha extrapolado para determinar, aunque sea de manera indirecta, la calidad de esa investigación publicada. Considerando únicamente el número absoluto de citas recibidas por las publicaciones realizadas entre 2002 y 2010, la universidad que

Tabla V. Productividad de las universidades públicas (10 primeras universidades). [Ver tabla completa]

\begin{tabular}{ccccccccccccc}
\hline Orden & Universidad & $\mathbf{2 0 0 2}$ & $\mathbf{2 0 0 3}$ & $\mathbf{2 0 0 4}$ & $\mathbf{2 0 0 5}$ & $\mathbf{2 0 0 6}$ & $\mathbf{2 0 0 7}$ & $\mathbf{2 0 0 8}$ & $\mathbf{2 0 0 9}$ & $\mathbf{2 0 1 0}$ & $\begin{array}{c}\text { Prom. docs. } \\
\text { /prom. Prof. }\end{array}$ & $\begin{array}{c}\text { Total } \\
\text { doc }\end{array}$ \\
\hline $\mathbf{1}$ & UPF & 0,63 & 0,87 & 1,01 & 1,04 & 1,43 & 1,78 & 2,07 & 2,11 & 2,31 & 1,47 & 3743 \\
\hline $\mathbf{2}$ & UAB & 0,67 & 0,69 & 0,82 & 0,93 & 1,04 & 1,36 & 1,30 & 1,41 & 1,44 & 1,07 & 13989 \\
\hline $\mathbf{3}$ & UMH & 0,79 & 0,80 & 0,77 & 0,83 & 0,86 & 0,91 & 1,05 & 1,04 & 1,03 & 0,88 & 2856 \\
\hline $\mathbf{4}$ & URV & 0,56 & 0,60 & 0,69 & 0,76 & 0,89 & 1,03 & 1,01 & 1,12 & 1,29 & 0,88 & 4041 \\
\hline $\mathbf{5}$ & UAM & 0,74 & 0,72 & 0,75 & 0,78 & 0,84 & 0,85 & 0,93 & 0,95 & 0,94 & 0,83 & 10957 \\
\hline $\mathbf{6}$ & UC3M & 0,88 & 0,90 & 0,77 & 0,84 & 0,89 & 0,75 & 0,77 & 0,93 & 0,91 & 0,82 & 3618 \\
\hline $\mathbf{7}$ & UB & 0,63 & 0,61 & 0,65 & 0,68 & 0,78 & 0,85 & 0,95 & 1,03 & 1,03 & 0,80 & 19400 \\
\hline $\mathbf{8}$ & USC & 0,63 & 0,69 & 0,60 & 0,63 & 0,74 & 0,74 & 0,78 & 0,80 & 0,81 & 0,71 & 9953 \\
\hline $\mathbf{9}$ & UJI & 0,62 & 0,65 & 0,71 & 0,71 & 0,72 & 0,64 & 0,69 & 0,73 & 0,78 & 0,69 & 2860 \\
\hline $\mathbf{1 0}$ & UPC & 0,45 & 0,51 & 0,59 & 0,64 & 0,70 & 0,74 & 0,85 & 0,86 & 0,92 & 0,69 & 8660 \\
\hline
\end{tabular}

Tabla VI. Porcentaje de documentos de las universidades públicas en colaboración internacional (10 primeras universidades). [Ver tabla completa]

\begin{tabular}{cccccccccccccc}
\hline Orden & Universidad & $\mathbf{2 0 0 2}$ & $\mathbf{2 0 0 3}$ & $\mathbf{2 0 0 4}$ & $\mathbf{2 0 0 5}$ & $\mathbf{2 0 0 6}$ & $\mathbf{2 0 0 7}$ & $\mathbf{2 0 0 8}$ & $\mathbf{2 0 0 9}$ & $\mathbf{2 0 1 0} \begin{array}{c}\text { Prom. } \\
\mathbf{\%}\end{array}$ & $\begin{array}{c}\text { Total doc } \\
\text { col. int }\end{array}$ \\
\hline $\mathbf{1}$ & UPF & 51,87 & 55,13 & 53,49 & 53,40 & 52,17 & 51,81 & 50,27 & 48,76 & 46,63 & 50,68 & 1897 \\
\hline $\mathbf{2}$ & UB & 40,50 & 41,28 & 43,33 & 44,49 & 48,05 & 46,70 & 46,98 & 48,06 & 48,88 & 45,89 & 8903 \\
\hline $\mathbf{3}$ & UAM & 43,56 & 43,53 & 43,98 & $39,8)$ & 39,89 & 42,98 & 42,66 & 44,68 & 45,73 & 43,09 & 4721 \\
\hline $\mathbf{4}$ & UAB & 37,93 & 38,94 & 39,02 & 42,17 & 44,54 & 41,72 & 43,77 & 44,80 & 45,50 & 42,70 & 5973 \\
\hline $\mathbf{5}$ & UPC & 40,94 & 41,76 & 40,10 & 41,48 & 40,96 & 40,72 & 44,20 & 43,15 & 44,20 & 42,17 & 3652 \\
\hline $\mathbf{6}$ & ULL & 33,43 & 35,14 & 37,98 & 39,00 & 37,02 & 40,66 & 39,12 & 46,29 & 55,67 & 41,87 & 1901 \\
\hline $\mathbf{7}$ & UIB & 46,57 & 35,44 & 43,46 & 44,44 & 43,97 & 36,99 & 39,07 & 41,11 & 42,51 & 41,42 & 1059 \\
\hline $\mathbf{8}$ & UDG & 26,79 & 39,68 & 43,35 & 40,07 & 46,05 & 47,68 & 41,64 & 37,50 & 42,68 & 41,26 & 1114 \\
\hline $\mathbf{9}$ & UV & 37,25 & 37,10 & 39,54 & 39,98 & 38,19 & 39,70 & 38,78 & 39,10 & 40,74 & 39,07 & 4939 \\
\hline $\mathbf{1 0}$ & URV & 35,11 & 36,60 & 40,06 & 35,86 & 39,23 & 38,54 & 39,02 & 40,56 & 39,13 & 38,55 & 1558 \\
\hline
\end{tabular}


se ubica en primera posición es la de Barcelona (UB), seguida por la Complutense de Madrid (UCM) y la Autónoma de Barcelona (UAB). Sin embargo, cuando se relativiza este indicador al considerar el número de citas por profesor, la primera posición la ocupa la Universidad Pompeu Fabra (UPF) con 17,78 citas/profesor, seguida por la Universidad Autónoma de Barcelona (UAB) con 10,34, y la Universidad de Barcelona (UB) con 9,46 (tabla VII).

\section{Visibilidad de las publicaciones}

Para analizar la visibilidad de las publicaciones, se ha calculado el porcentaje de documentos en el primer cuartil de cada disciplina (Q1). Para el cálculo no se han considerado las publicaciones totales, sino sólo aquellas publicaciones en revistas incluidas en el JCR. En este sentido, hay que señalar que no existe un JCR para algunas de las disciplinas de humanidades, por estar incluidas en A\&HCI, aunque otras, como historia, antropología o lingüística, también se encuentran recogidas en el JCR/SSCI. Sin embargo, en general, y por las características que presenta el trabajo de los investigadores en humanidades, gran parte de su producción científica va a tener una escasa visibilidad internacional, lo que indudablemente perjudica a las instituciones que tengan fortalezas en estas áreas.

Los datos muestran que el porcentaje de revistas en el primer cuartil (Q1) varía entre el 36,91\% y el $58,55 \%$. Si se consideran las 3 primeras instituciones con mayor porcentaje de documentos en el cuartil de mayor visibilidad, destacan la Universidad Pompeu Fabra (UPF) con el 58,55\%, la Universidad de Córdoba (UCO) con el 58,49\%, y la Universidad de Barcelona (UB) con el 57,27\%. En valores absolutos, por número de documentos publicados en el primer cuartil, permanecerían 2 de las 3 universidades con porcentajes más elevados, la UB en primer lugar con un total de 10.556 documentos publicados en revistas de este cuartil, y la $U A B$ en tercer lugar con 7.331 documentos, mientras que la Universidad Complutense de Madrid (UCM) se colocaría en segunda posición con 8.185 documentos (tabla VIII)

Tabla VII. Citas por profesor de las universidades públicas (10 primeras universidades por promedio de citas/profesor). [Ver tabla completa]

\begin{tabular}{|c|c|c|c|c|c|c|c|c|c|c|c|c|}
\hline Orden & Universidad & 2002 & 2003 & 2004 & 2005 & 2006 & 2007 & 2008 & 2009 & 2010 & $\begin{array}{l}\text { Promedio } \\
\text { citas/prof. }\end{array}$ & $\begin{array}{l}\text { Total } \\
\text { citas }\end{array}$ \\
\hline 1 & UPF & 19,20 & 16,95 & 20,67 & 19,48 & 24,24 & 24,45 & 19,12 & 11,34 & 4,66 & 17,78 & 45367 \\
\hline 2 & UAB & 11,08 & 12,55 & 11,84 & 14,03 & 13,49 & 13,04 & 8,91 & 6,01 & 2,63 & 10,34 & 134778 \\
\hline 3 & UB & 13,08 & 13,36 & 11,68 & 11,07 & 10,50 & 9,60 & 8,34 & 5,43 & 2,14 & 9,46 & 229055 \\
\hline 4 & UAM & 16,07 & 14,01 & 13,60 & 11,99 & 9,81 & 9,45 & 6,70 & 4,20 & 2,03 & 9,23 & 121966 \\
\hline 5 & URV & 10,73 & 10,31 & 10,52 & 11,26 & 12,53 & 10,31 & 8,64 & 5,81 & 2,34 & 9,18 & 42074 \\
\hline 6 & UMH & 17,74 & 11,11 & 10,81 & 10,81 & 10,90 & 9,05 & 6,21 & 3,82 & 1,54 & 8,28 & 26828 \\
\hline 7 & USC & 9,46 & 9,22 & 8,75 & 8,47 & 8,42 & 6,13 & 5,57 & 3,71 & 1,64 & 6,62 & 92800 \\
\hline 8 & UV & 10,21 & 8,67 & 8,72 & 8,16 & 8,20 & 5,72 & 5,26 & 3,53 & 1,44 & 6,60 & 133928 \\
\hline 9 & UJI & 9,24 & 9,44 & 9,39 & 9,35 & 7,29 & 5,64 & 5,30 & 3,75 & 1,53 & 6,46 & 26836 \\
\hline 10 & UDG & 6,15 & 7,67 & 9,57 & 8,31 & 8,06 & 6,65 & 5,24 & 3,44 & 1,79 & 6,11 & 24539 \\
\hline
\end{tabular}

Tabla VIII. Publicaciones en revistas del Q1 de las universidades públicas. (10 primeras universidades por porcentaje de publicaciones en Q1). [Ver tabla completa]

\begin{tabular}{cccccccccccccc}
\hline Orden & Universidad & $\mathbf{2 0 0 2}$ & $\mathbf{2 0 0 3}$ & $\mathbf{2 0 0 4}$ & $\mathbf{2 0 0 5}$ & $\mathbf{2 0 0 6}$ & $\mathbf{2 0 0 7}$ & $\mathbf{2 0 0 8}$ & $\mathbf{2 0 0 9}$ & $\mathbf{2 0 1 0}$ & $\begin{array}{c}\text { Total } \\
\mathbf{0}\end{array}$ & $\begin{array}{c}\text { Total doc } \\
\mathbf{Q 1}\end{array}$ \\
\hline $\mathbf{1}$ & UPF & 62,15 & 51,46 & 55,28 & 54,35 & 60,74 & 61,50 & 64,15 & 55,78 & 58,43 & 58,55 & 1996 \\
\hline $\mathbf{2}$ & UCO & 58,15 & 53,77 & 56,23 & 57,65 & 58,90 & 60,16 & 63,18 & 57,67 & 57,89 & 58,49 & 2417 \\
\hline $\mathbf{3}$ & UB & 57,82 & 55,12 & 56,59 & 57,11 & 59,50 & 59,03 & 55,91 & 57,63 & 56,45 & 57,27 & 10556 \\
\hline $\mathbf{4}$ & UBU & 58,82 & 59,15 & 40,43 & 57,55 & 67,42 & 61,65 & 53,60 & $50,4)$ & 56,43 & 56,03 & 539 \\
\hline $\mathbf{5}$ & UAB & 50,77 & 54,06 & 49,70 & 53,01 & 57,98 & 59,13 & 55,80 & $56,9)$ & 57,87 & 55,67 & 7331 \\
\hline $\mathbf{6}$ & UAM & 56,85 & 53,33 & 52,60 & 57,44 & 53,87 & 56,82 & 51,11 & 56,14 & $55,4)$ & 54,85 & 5577 \\
\hline $\mathbf{7}$ & URV & 48,67 & 54,51 & 51,58 & 48,53 & 56,64 & 54,79 & 57,46 & 57,43 & 56,24 & 54,60 & 2094 \\
\hline $\mathbf{8}$ & UDL & 51,11 & 53,90 & 51,96 & 51,98 & 53,42 & 59,91 & 55,43 & 52,75 & 53,96 & 54,02 & 1082 \\
\hline $\mathbf{9}$ & UIB & 56,57 & 43,68 & 53,73 & 49,35 & 52,87 & 57,20 & 52,26 & 59,13 & 53,50 & 53,55 & 1283 \\
\hline $\mathbf{1 0}$ & UDG & 47,71 & 41,57 & 43,50 & 46,54 & 50,37 & 50,35 & 60,23 & 55,81 & 58,17 & 1327 & 1327 \\
\hline
\end{tabular}




\subsection{Dimensión 4: Innovación}

La inclusión de esta dimensión es novedosa con respecto a otros rankings, ya que se presentan datos sobre patentes, ingresos obtenidos por contratos y consultorías, número de extensiones PCT y creación de spin off. Exceptuando la información sobre patentes, todos los datos son recogidos por una encuesta anual que realiza la Red OTRI. En la tabla IX se muestran las universidades públicas según el número de patentes concedidas por cada 100 profesores durante el período analizado. Como se puede apreciar en la tabla, en las 2 primeras posiciones se sitúan instituciones con un perfil tecnológico, como son la Universidad Politécnica de Cataluña (UPC) y la Politécnica de Valencia (UPV), seguidas de universidades con perfil más generalista, como las universidades de Navarra, Burgos y Cantabria. Sin embargo, si se consideran valores absolutos, a las 2 universidades politécnicas mencionadas se une en tercer lugar la Universidad Politécnica de Madrid (UPM).

\subsection{Dimensión 5: Competitividad}

Otra dimensión importante es la capacidad de las universidades para la captación de fondos dedicados a la investigación. Esta dimensión se puede determinar a través de varios indicadores, como el número de proyectos nacionales y europeos obtenidos en convocatorias competitivas. En este sentido, en la tabla $X$ se puede observar que en las primeras posiciones del ranking, en cuanto a número de proyectos obtenidos en convocatorias del Plan Nacional por cada 100 profesores, se ubican instituciones jóvenes y de pequeño tamaño, como son la Universidad Pompeu Fabra (UPF), la Universidad Pablo de Olavide (UPO), la Universidad Carlos III de Madrid (UC3M) y la Universidad de Girona (UDG).

Cuando se considera la participación en proyectos europeos, concretamente en las convocatorias de los Programas Marco, entre las 4 primeras universidades, 2 de ellas ocupan las mismas posiciones que en las convocatorias del Plan Nacional, la

Tabla IX. Número de patentes concedidas por las universidades públicas (10 primeras universidades). [Ver tabla completa]

\begin{tabular}{ccccccccccccc}
\hline Orden & Universidad & $\mathbf{2 0 0 2}$ & $\mathbf{2 0 0 3}$ & $\mathbf{2 0 0 4}$ & $\mathbf{2 0 0 5}$ & $\mathbf{2 0 0 6}$ & $\mathbf{2 0 0 7}$ & $\mathbf{2 0 0 8}$ & $\mathbf{2 0 0 9}$ & $\mathbf{2 0 1 0}$ & $\begin{array}{c}\text { Total patentes } \\
\text { / 100 prof }\end{array}$ & $\begin{array}{c}\text { Total } \\
\text { patentes }\end{array}$ \\
\hline $\mathbf{1}$ & UPC & 0,58 & 1,89 & 1,21 & 1,50 & 1,97 & 2,14 & 1,24 & 1,66 & 3,22 & 1,70 & 214 \\
\hline $\mathbf{2}$ & UPV & 0,55 & 1,80 & 1,44 & 1,96 & 1,16 & 1,72 & 0,72 & 0,92 & 1,02 & 1,25 & 197 \\
\hline $\mathbf{3}$ & UNAVARRA & 0,84 & 1,40 & 0,55 & 0,82 & 1,08 & 1,54 & 0,50 & 0,95 & 0,93 & 0,95 & 33 \\
\hline $\mathbf{4}$ & UBU & 0,28 & 1,11 & 1,05 & 0,79 & 1,07 & 1,06 & 1,05 & 1,32 & 0,76 & 0,95 & 32 \\
\hline $\mathbf{5}$ & UNICAN & 0,64 & 0,16 & 0,79 & 0,80 & 0,64 & 0,62 & 1,23 & 1,98 & 1,53 & 0,94 & 54 \\
\hline $\mathbf{6}$ & UVIGO & 0,11 & 0,59 & 1,10 & 0,85 & 0,89 & 0,86 & 1,17 & 1,28 & 0,49 & 0,82 & 70 \\
\hline $\mathbf{7}$ & USC & 0,57 & 0,42 & 0,66 & 1,04 & 1,14 & 0,44 & 0,88 & 0,97 & 0,94 & 0,79 & 112 \\
\hline $\mathbf{8}$ & UMH & 0,77 & 0,00 & 1,56 & 1,25 & 0,84 & 0,27 & 0,00 & 1,26 & 0,94 & 0,76 & 24 \\
\hline $\mathbf{9}$ & UAH & 0,13 & 1,14 & 0,38 & 0,36 & 0,82 & 1,04 & 0,23 & 1,39 & 1,29 & 0,76 & 63 \\
\hline $\mathbf{1 0}$ & UAL & 0,26 & 0,73 & 0,21 & 0,77 & 0,94 & 0,74 & 0,69 & 0,86 & 1,21 & 0,74 & 34 \\
\hline
\end{tabular}

Tabla X. Número de proyectos de las universidades públicas obtenidos en convocatorias del Plan Nacional por 100 profesores (10 primeras universidades). [Ver tabla completa]

\begin{tabular}{ccccccccccccc}
\hline Orden & Universidad & $\mathbf{2 0 0 2}$ & $\mathbf{2 0 0 3}$ & $\mathbf{2 0 0 4}$ & $\mathbf{2 0 0 5}$ & $\mathbf{2 0 0 6}$ & $\mathbf{2 0 0 7}$ & $\mathbf{2 0 0 8}$ & $\mathbf{2 0 0 9}$ & $\mathbf{2 0 1 0}$ & $\begin{array}{c}\text { Total proy. PN/ } \\
\mathbf{1 0 0} \text { profesores }\end{array}$ & $\begin{array}{c}\text { Total } \\
\text { PN }\end{array}$ \\
\hline $\mathbf{1}$ & UPF & 8,36 & 15,23 & 10,70 & 9,54 & 16,06 & 10,23 & 12,78 & 17,77 & 13,90 & 12,56 & 327 \\
\hline $\mathbf{2}$ & UPO & 11,11 & 9,43 & 8,06 & 10,16 & 13,10 & 9,50 & 7,60 & 6,12 & 5,69 & 10,51 & 135 \\
\hline $\mathbf{3}$ & UC3M & 10,51 & 10,83 & 10,83 & 10,02 & 10,11 & 7,56 & 8,71 & 9,00 & 7,53 & 10,14 & 394 \\
\hline $\mathbf{4}$ & UDG & 7,58 & 6,87 & 7,06 & 7,26 & 10,00 & 8,71 & 7,38 & 8,09 & 7,35 & 8,13 & 308 \\
\hline $\mathbf{5}$ & UAB & 6,46 & 8,20 & 5,57 & 7,21 & 9,27 & 8,47 & 6,70 & 9,49 & 8,59 & 7,84 & 1012 \\
\hline $\mathbf{6}$ & UAM & 7,45 & 6,67 & 7,51 & 8,45 & 7,00 & 5,09 & 8,34 & 7,46 & 5,50 & 7,40 & 913 \\
\hline $\mathbf{7}$ & UMH & 9,96 & 5,94 & 5,30 & 10,00 & 5,04 & 3,27 & 8,29 & 6,03 & 4,46 & 6,77 & 198 \\
\hline $\mathbf{8}$ & UJI & 8,51 & 4,92 & 6,09 & 5,80 & 7,03 & 5,47 & 7,46 & 5,17 & 4,50 & 6,37 & 248 \\
\hline $\mathbf{9}$ & UB & 6,72 & 5,99 & 5,15 & 7,15 & 7,10 & 4,83 & 6,92 & 7,44 & 5,60 & 6,33 & 1528 \\
\hline $\mathbf{1 0}$ & UNICAN & 7,41 & 4,10 & 5,71 & 7,80 & 6,53 & 5,09 & 6,76 & 5,65 & 6,60 & 6,24 & 355 \\
\hline
\end{tabular}


Universidad Pompeu Fabra (UPF) y la Universidad Carlos III (UC3M) (tabla XI). En proyectos europeos aparecen en segundo y cuarto lugar las universidades Politécnica de Catalunya (UPC) y la Rovira y Virgili (URV), que no se encontraba entre las 10 primeras por su actividad en proyectos del Plan Nacional. En sentido inverso, la Universidad Pablo de Olavide (UPO) y la Universidad de Girona (UDG) pasan de la segunda y cuarta posición en proyectos nacionales a la decimocuarta y decimosegunda respectivamente en los del Programa Marco.

\subsection{Dimensión 6: Capacidad Formativa para la investigación}

Otras actividades científicas importantes dentro del ámbito universitario están relacionadas con la formación de investigadores y con la atracción de talento. En este sentido, el número de becas, contratos postdoctorales, y la defensa de tesis doctorales constituyen indicadores de gran interés para conocer la capacidad de las universidades en esta dimensión. En este trabajo solo se muestran los datos de las tesis defendidas relativas al número de profesores. Como se aprecia en la tabla XII, considerando los valores de este indicador destaca en primer lugar la Universidad Autónoma de Madrid (UAM), seguida de la Autónoma de Barcelona (UAB) y la Pompeu Fabra (UPF).

\subsection{Relación entre dimensiones}

Con los datos presentados se ha podido observar la posición que adquiere cada universidad en las distintas dimensiones analizadas. Estos datos permiten comparar, por ejemplo, la proporción de documentos que cada universidad representa sobre el total del sistema universitario, frente a la proporción que representan sus citas. De esta forma se aprecia que algunas instituciones cuentan con un impacto mayor del esperado, como es el caso de la Universidad de Barcelona (UB), la de Valencia (UV), la Autónoma de Madrid (UAM) o la Pompeu Fabra (UPF), donde el porcentaje de citas supera al de documentos publicados. En otras, por el contrario, la proporción de citas es mucho menor

Tabla XI. Número de proyectos de las universidades públicas obtenidos en convocatorias del Programa Marco de la UE por 100 profesores (10 primeras universidades). [Ver tabla completa]

\begin{tabular}{ccccccccccccc}
\hline Orden & Universidad & $\mathbf{2 0 0 2}$ & $\mathbf{2 0 0 3}$ & $\mathbf{2 0 0 4}$ & $\mathbf{2 0 0 5}$ & $\mathbf{2 0 0 6}$ & $\mathbf{2 0 0 7}$ & $\mathbf{2 0 0 8}$ & $\mathbf{2 0 0 9}$ & $\mathbf{2 0 1 0}$ & $\begin{array}{c}\text { Total PM/ } \\
\mathbf{1 0 0} \text { prof. }\end{array}$ & \begin{tabular}{c} 
Total PM \\
\hline $\mathbf{1}$
\end{tabular} \\
\hline UPF & 2,34 & 3,97 & 1,67 & 3,18 & 4,74 & 4,17 & 4,51 & 4,53 & 7,46 & 4,05 & 104 \\
\hline $\mathbf{2}$ & UPC & 1,96 & 2,38 & 1,28 & 2,21 & 2,26 & 1,33 & 1,32 & 1,52 & 1,87 & 1,79 & 224 \\
\hline $\mathbf{3}$ & UC3M & 3,60 & 3,89 & 0,76 & 1,82 & 1,47 & 1,51 & 1,27 & 0,69 & 1,00 & 1,62 & 69 \\
\hline $\mathbf{4}$ & URV & 1,40 & 1,77 & 1,55 & 2,38 & 0,76 & 0,99 & 1,39 & 1,56 & 2,80 & 1,62 & 74 \\
\hline $\mathbf{5}$ & UAB & 0,78 & 1,38 & 1,17 & 0,88 & 0,76 & 1,18 & 1,57 & 1,42 & 2,23 & 1,27 & 165 \\
\hline $\mathbf{6}$ & UPM & 1,37 & 1,80 & 1,02 & 1,13 & 1,20 & 1,26 & 1,23 & 1,17 & 1,21 & 1,26 & 282 \\
\hline $\mathbf{7}$ & UMH & 3,45 & 1,25 & 0,93 & 1,25 & 0,56 & 0,82 & 1,34 & 0,75 & 0,47 & 1,11 & 35 \\
\hline $\mathbf{8}$ & UPV & 0,92 & 1,02 & 0,63 & 0,86 & 0,75 & 0,52 & 0,94 & 1,19 & 0,86 & 0,86 & 135 \\
\hline $\mathbf{9}$ & UNICAN & 1,29 & 1,10 & 0,32 & 1,27 & 0,48 & 0,77 & 0,77 & 0,76 & 0,92 & 0,85 & 49 \\
\hline $\mathbf{1 0}$ & UB & 0,83 & 1,21 & 0,68 & 0,51 & 0,59 & 0,48 & 0,74 & 0,82 & 0,72 & 0,73 & 176 \\
\hline
\end{tabular}

Tabla XII. Número de tesis defendidas por las universidades públicas por 100 profesores (10 primeras universidades). [Ver tabla completa]

\begin{tabular}{|c|c|c|c|c|c|c|c|c|c|c|c|c|}
\hline Orden & Universidad & 2002 & 2003 & 2004 & 2005 & 2006 & 2007 & 2008 & 2009 & 2010 & $\begin{array}{c}\text { Total tesis/ } \\
100 \text { prof }\end{array}$ & $\begin{array}{l}\text { Total } \\
\text { tesis }\end{array}$ \\
\hline 1 & UAM & 24.48 & 29.07 & 31.55 & 52.61 & 20.45 & 25.41 & 22.46 & 24.67 & 28.80 & 28.66 & 3726 \\
\hline 2 & UAB & 20.81 & 20.19 & 21.31 & 21.84 & 21.29 & 28.52 & 25.77 & 31.03 & 31.66 & 24.72 & 3217 \\
\hline 3 & UPF & 12.71 & 11.92 & 12.71 & 15.90 & 20.80 & 22.35 & 29.70 & 32.40 & 40.00 & 21.92 & 563 \\
\hline 4 & UMH & 14.94 & 17.81 & 23.05 & 24.06 & 19.33 & 17.17 & 19.52 & 18.34 & 16.43 & 18.92 & 595 \\
\hline 5 & UB & 18.45 & 18.23 & 17.03 & 17.52 & 18.88 & 18.49 & 19.82 & 19.93 & 19.99 & 18.70 & 4522 \\
\hline 6 & UCM & 18.61 & 18.22 & 18.31 & 24.58 & 20.21 & 15.45 & 15.29 & 16.59 & 17.86 & 18.30 & 6284 \\
\hline 7 & USAL & 10.94 & 15.29 & 12.27 & 42.15 & 12.95 & 15.66 & 18.03 & 17.06 & 16.19 & 17.88 & 2198 \\
\hline 8 & UCO & 13.30 & 16.69 & 24.38 & 13.47 & 15.69 & 15.78 & 15.17 & 15.75 & 24.88 & 17.26 & 1247 \\
\hline 9 & UPO & 15.28 & 4.72 & 10.48 & 15.62 & 19.31 & 22.50 & 19.20 & 21.77 & 14.37 & 17.06 & 282 \\
\hline 10 & UC3M & 14.71 & 18.33 & 19.40 & 14.81 & 12.84 & 17.77 & 14.52 & 14.36 & 21.24 & 16.48 & 702 \\
\hline
\end{tabular}


a lo esperado como ocurre en las universidades politécnicas (figura 1 ).

A partir de los datos obtenidos, se ha observado también la existencia de correlación entre la competitividad -medida a través del número de proyectos obtenidos en las convocatorias de los Programa Marco- y la productividad de las universidades $\left(R^{2}=0,63\right)$. En este sentido, se puede decir que en el $63 \%$ de los casos la productividad de las universidades ha estado relacionada con su competitividad a la hora de obtener proyectos en las convocatorias del Programa Marco. Tal como se puede observar en la figura 2, existe un grupo de universidades con valores elevados en ambas variables. Entre ellas destaca específicamente la Universidad Pompeu Fabra (UPF) y completan el grupo la Autónoma de Barcelona (UAB), la Miguel Hernández (UMH), la Rovira i Virgili (URV), y la Carlos
III (UC3M). Evidentemente, el hecho de que algunas universidades hayan seguido una política de profesorado más restrictiva a la hora de consolidar sus plantillas con profesorado permanente afecta a los cálculos de su actividad investigadora, y de ahí que en muchos casos su posición destaque del resto de universidades.

En la figura 2 se muestra también una tercera variable, que es el número de citas por profesor, representada mediante el tamaño de las burbujas. A partir de esta variable se observa una clara relación entre el impacto relativo y la productividad de las universidades $\left(R^{2}=0,90\right)$. Este resultado mostraría claramente la dependencia existente entre estos dos indicadores, que de algún modo son resultados que se han ido viendo en los distintos indicadores unidimensionales de la actividad científica mostrados.

Figura 1. Proporción de documentos y citas en cada universidad con respecto al total del sistema

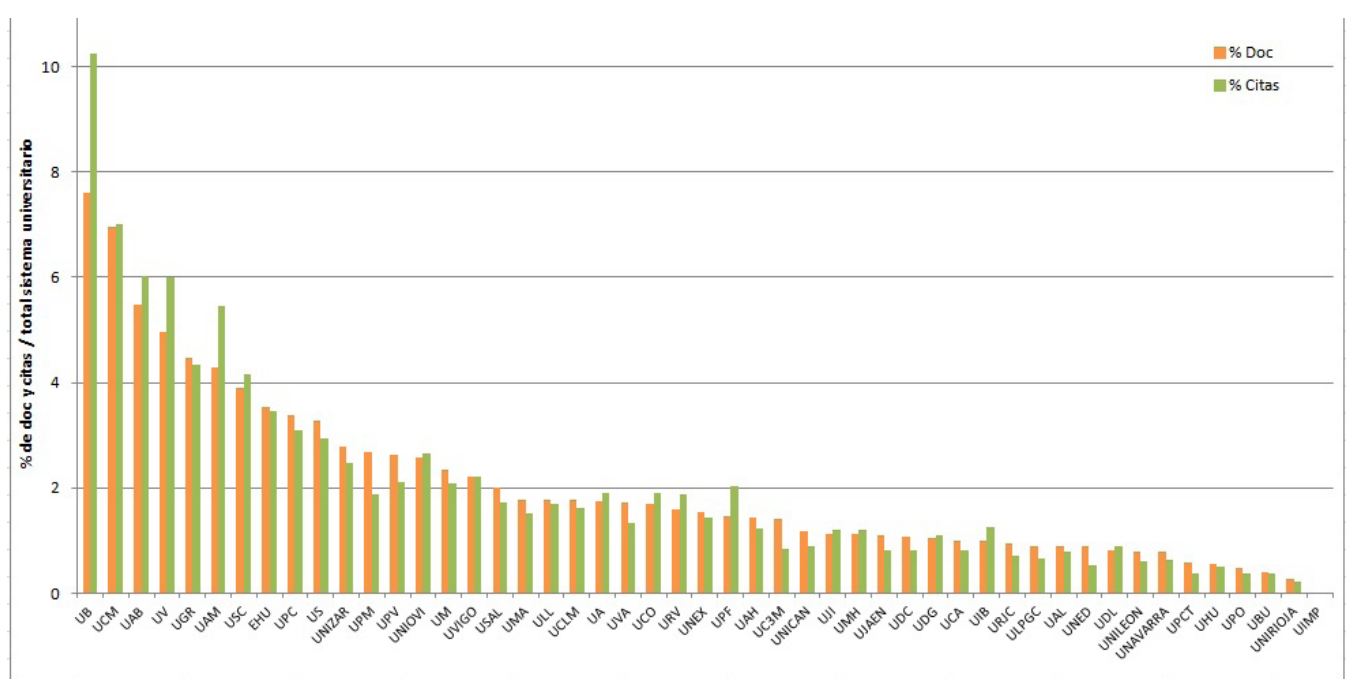

Figura 2. Productividad, competitividad e impacto (tamaño de las burbujas) de las universidades

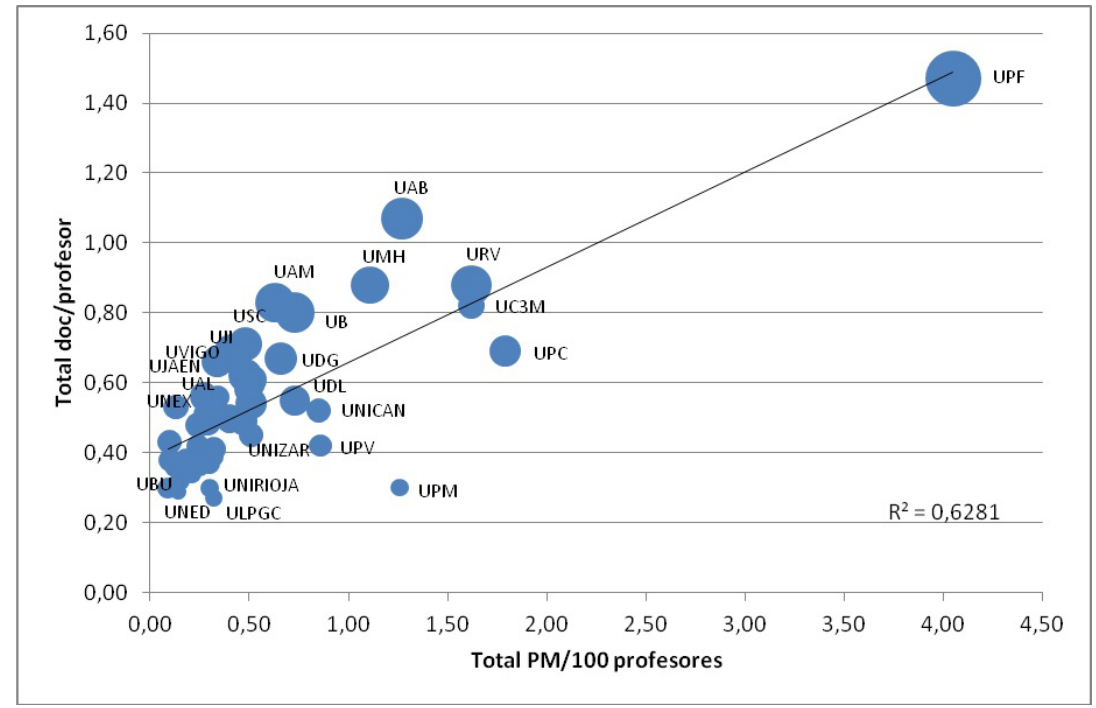


El hecho de mostrar de manera separada la posición de las universidades en las diferentes dimensiones permite observar los distintos perfiles de cada una de ellas. En la figura 3 se puede observar claramente la ausencia de relación entre la actividad publicadora en revistas internacionales y la actividad patentadora $\left(R^{2}=0,0084\right)$. Las universidades de perfil tecnológico que aparecen con una intensa actividad patentadora tanto en valores absolutos como relativos al profesorado, como son la Universidad Politécnica de Cataluña (UPC) y la Universidad Politécnica de Valencia (UPV), muestran una menor actividad publicadora que otras universidades de perfil más generalista como son la Universidad Pompeu Fabra (UPF) y la Universidad Autónoma de Barcelona (UAB). Como se ha comentado a lo largo del trabajo, la orientación de la actividad científica de las universidades estaría afectando los datos obtenidos de determinados indicadores, como es el caso de la actividad publicadora.

\section{DISCUSIÓN Y CONCLUSIONES}

El continuo desarrollo y la creación de nuevos rankings para evaluar la calidad de las universidades está generando un intenso debate sobre su utilidad real debido a la aparente contradicción que existe en muchos de ellos, ya que la obtención de información con fuentes y metodologías diferentes provoca en ocasiones que los resultados obtenidos sean muy distintos. A pesar de ello, es imposible no reconocer el éxito que tienen, puesto que, como resultado de la globalización de la educación superior, las universidades deben competir internacionalmente por recursos económicos y humanos, y por lo tanto suelen utilizar los rankings como herramienta para promocionar y dar a conocer su excelencia tanto en docencia como en investigación (Aguillo y otros, 2010). Desde el punto de vista de los potenciales "usuarios" de las universidades (alumnos, profesores, gestores académicos) como de los evaluadores de las actividades de I+D, los rankings también adquieren gran utilidad, ya que ofrecen información sobre diversos aspectos de la actividad universitaria, que puede resultar esencial tanto a la hora de evaluar la productividad como para conocer aspectos ligados a la orientación o a factores generales que den cuenta del prestigio y reconocimiento de una institución. Todos estos elementos que influyen sobre el posicionamiento de una universidad tienen un impacto directo sobre su imagen y, por lo tanto, la elaboración de rankings debe ser abordada con responsabilidad y siguiendo unas normativas precisas que faciliten la transparencia, la objetividad y el rigor de las clasificaciones (Buela-Casal y otros, 2010). Asimismo, es fundamental recordar que pueden desarrollarse otras iniciativas que coexistan con los rankings y que permitan poner de relieve la diversidad de cada

Figura 3. Productividad y patentes por profesor

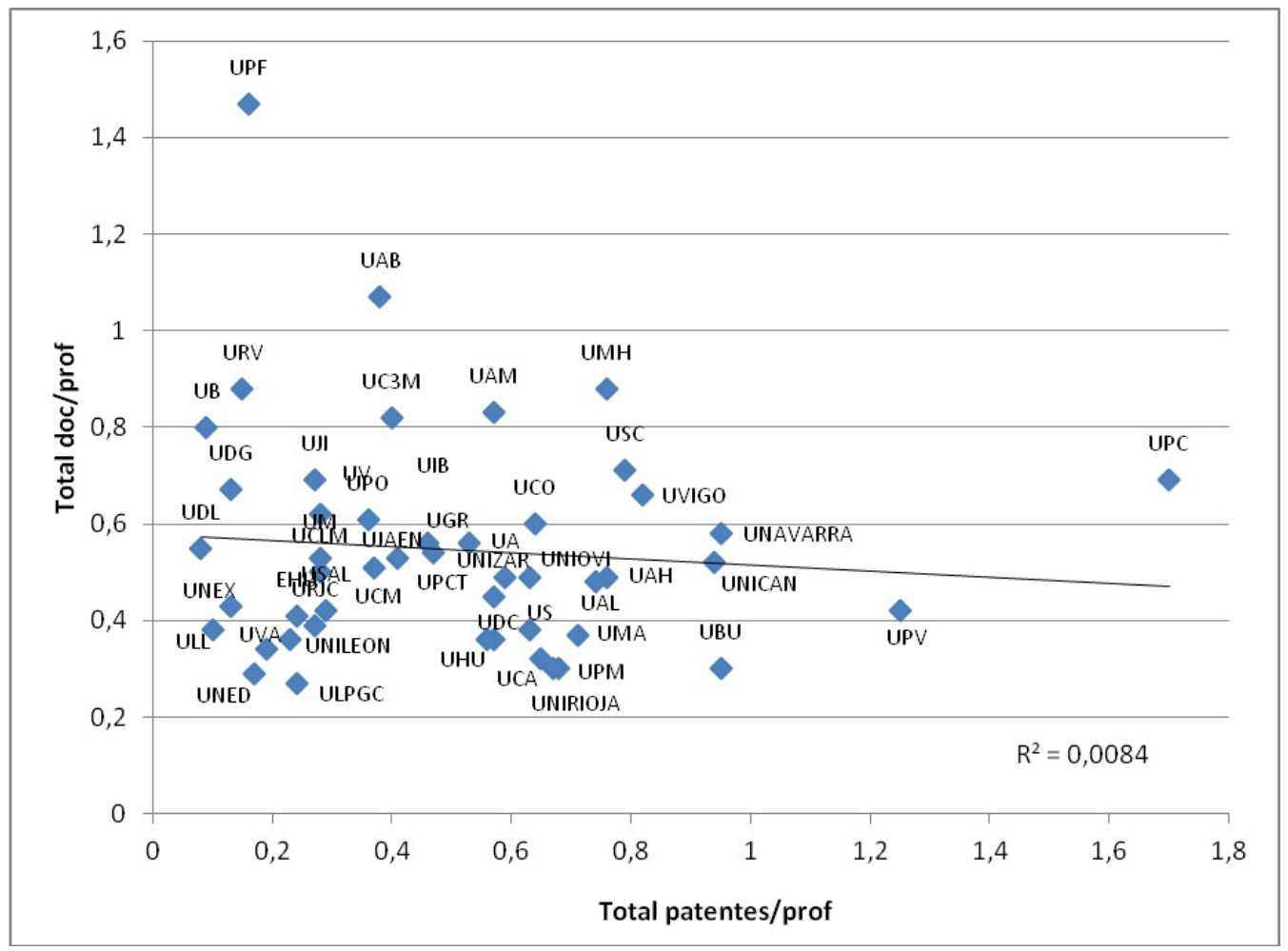


sistema universitario y a su vez realizar comparaciones entre instituciones de un mismo entorno. Teniendo en cuenta estas consideraciones, el Observatorio IUNE puede contemplar las directrices que deben seguir las clasificaciones de universidades, tanto en el contexto internacional como en el español. Por ello, adopta los principios de Berlín para la creación de Rankings de Instituciones de Educación superior (International Ranking Expert Group, 2006).

Desde el punto de vista metodológico, las ventajas que presenta el Observatorio se centran en la utilización de fuentes oficiales, lo que ofrece transparencia y permite a los usuarios constatar la información utilizada. La asignación de registros a las instituciones correspondientes es también un factor esencial en el que el Observatorio IUNE ha demostrado un alto grado de precisión. Esto es fundamental, dado que muchas veces se ha detectado una incorrecta desagregación de datos (considerar, por ejemplo, a ciertos institutos universitarios de manera independiente o no reconocer siglas o direcciones postales de departamentos o facultades) ha llevado a atomizar la información relativa a una institución y modificar notablemente su posición en un ranking (van-Raan, 2005).

Otro punto clave del Observatorio es la inclusión de 6 dimensiones que permiten conocer diversos aspectos de la actividad investigadora realizada en las universidades, como la transferencia o la competitividad, que son elementos esenciales a la hora de analizar la calidad de las universidades. No centrarse únicamente en la producción científica - como suele ocurrir con la mayor parte de los rankings (Buesa y otros, 2009)- permite detectar un abanico mucho más amplio de actividades de cada universidad. La desagregación de la información, considerando separadamente a las universidades públicas y privadas cobra también gran relevancia, ya que sus diferentes orientaciones hacen que en la mayoría de los indicadores los resultados obtenidos no sean comparables. En general, gran parte de las universidades públicas son multidisciplinares, y cuentan con un número considerablemente más alto de cátedras y alumnos que las privadas (el $91 \%$ de alumnos corresponde a las universidades públicas y el $9 \%$ a las privadas) (Bordons y otros, 2010). Asimismo, la posibilidad de obtener diferentes rankings en función del indicador y la dimensión analizada ofrece mayor flexibilidad y menor reduccionismo, permitiendo detectar los diferentes perfiles de actividad en cada una de las universidades.

Respecto a los resultados obtenidos en este trabajo, la información sobre profesorado muestra que en promedio, las 10 universidades públicas de mayor tamaño (UCM, UB, UPM, EHU, US, UV, UGR, UPV, UNIZAR y USC) concentran el $43 \%$ del profesorado permanente del sistema universitario público español ${ }^{1}$. En los sexenios, es el profesorado de las universidades más jóvenes el que muestra una mayor capacidad en la obtención de este tipo de reconocimiento, ya que 4 de estas universidades (UPO, UC3M, UMH y UPF) se encuentran entre las 5 primeras posiciones. Resultados parecidos obtuvo Buela-Casal en el Ranking de investigación de las universidades públicas españolas (Buela-Casal y otros, 2010).

En relación a las publicaciones científicas, según se constata en estudios previos, el sector universitario es el que tiene una mayor actividad, ya que produce en torno a un $62 \%$ de las publicaciones incluidas en la base de datos WoS (Gómez y otros, 2006). Dentro de este sector existe un predominio de la actividad de las universidades públicas que puede explicarse por el hecho de que éstas están más orientadas a la investigación que las privadas, donde prima más la actividad docente. Éste es también el caso de las Escuelas de Ingeniería, que tradicionalmente han mostrado una mayor dedicación a la docencia y a resolver problemas técnicos para las empresas, que a la investigación (Bordons y otros, 2010).

Los indicadores obtenidos muestran, en general, un incremento importante de la producción de las universidades. Al final del período este incremento es del $91 \%$ respecto a 2002 , siendo algo superior al observado en los mismos años en el Rankings ISI de la universidad española en la WOS (TorresSalinas y otros, 2011a) que fue del $81,21 \%$. Este crecimiento observado no es exclusivo del sector universitario ya que tal como muestran otros estudios (Delgado-López-Cozar y otros, 2009) el aumento de la producción de España en el mundo es un fenómeno que alcanza a todos los campos científicos. Según los autores, una posible explicación es la política de incentivos a la internacionalización llevada a cabo por la CNEAI, junto a la progresiva incorporación de revistas españolas a las bases de datos del WoS auspiciada por la política desarrollada en la FECYT.

Según muestran los datos del Observatorio IUNE, cinco de las 10 universidades más productivas son jóvenes y de pequeño tamaño (UPF, UC3M, UMH, URV, UJI). Esto evidencia que el grado de relación esperable entre el número de profesores y el número de publicaciones no se cumple en muchas de las universidades más consolidadas. Prácticamente las mismas universidades son las que aparecen también como las más productivas en el Ranking de investigación de las universidades públicas españolas (Buela-Casal y otros, 2010). Sin embargo, con otro de los rankings, el Índice multidimensional de la calidad de las universidades (Buesa y otros, 2009), sólo coincide en una de las universidades mencionadas (URV), mientras el resto de instituciones se posiciona en lugares mucho más discretos en el índice.

La productividad estaría vinculada con la mayor orientación de la universidad hacia determinadas áreas de investigación, por ejemplo las politécnicas, que como se ha comentado presentan mayor 
colaboración con el ámbito empresarial y menor actividad publicadora en revistas incluidas en bases de datos con índices de impacto, así como con las áreas de ciencias sociales y humanidades cuyas publicaciones están muy mal recogidas en la WoS.

Las universidades más grandes son las que tienen un mayor impacto global (mayor volumen de citas recibidas). Sin embargo, cuando se analiza el impacto de las publicaciones relativizado al número de profesores, 3 de las universidades pequeñas aparecen entre las 6 de mayor impacto (UPF, URV, UMH). En este sentido, las 2 universidades más destacadas, la UPF y la UAB con más de 10 citas/ prof en el período estudiado, muestran valores muy por debajo de los recibidos por otras universidades europeas. Esta escasa citación de la producción de las universidades españolas puede deberse, como argumentan Torres-Salinas y otros (2011a), a un estancamiento en la presencia de publicaciones en la élite (Q1, TOP 3) lo que convierte a las publicaciones españolas en poco atractivas para ser citadas. Es evidente, además, una falta de relación entre el incremento de la producción total y el aún escaso porcentaje de citas recibidas. Esta situación ha sido también observada en otros estudios como el realizado por Gómez y otros (2009), cuyos autores plantean que, si bien el conjunto de la producción española ha crecido notablemente, pasando del puesto 15 del ranking mundial en 1982 al 10 en 2003 (lo que representa el 3\% del mundo), las citas recibidas por publicaciones españolas solo alcanzan el $0,7 \%$ del total mundial.

Los indicadores relacionados con visibilidad muestran que, en promedio, las universidades públicas cuentan con alrededor del $48 \%$ de sus publicaciones en revistas del primer cuartil. Las 5 de ellas que han alcanzado mayor visibilidad, al publicar más del $55 \%$ de los artículos en revistas del primer cuartil, son: UPF, UCO, UB, UBU y UAB. Cuando se relacionan las instituciones que han obtenido valores más elevados en el indicador de visibilidad con las de citas por profesor y en colaboración internacional, se observa que 6 de ellas están entre las 10 primeras por impacto relativo y 7 entre las que muestran un mayor porcentaje de documentos en colaboración internacional. Estos resultados vendrían a demostrar la relación existente entre estos 3 indicadores que tienen un mayor componente cualitativo.

Cuando se comparan los valores de visibilidad obtenidos en el Observatorio IUNE con otros rankings mencionados, se observa que el $90 \%$ de las universidades que están en las 10 primeras posiciones, en función de los porcentajes de publicaciones en el primer cuartil, son las mismas (UPF, UCO, UB, UBU, UAB, UAM, URV, UIB y UDG) que en el Ranking ISI de las universidades españolas (Torres Salinas y otros, 2011b).

En cuanto a los indicadores de Innovación, destacan en esta dimensión las universidades politécnicas o con perfil más tecnológico, mientras que las "generalistas" tienen mejores posiciones en los indicadores de actividad científica. Las universidades politécnicas de Cataluña (UPC) y de Valencia (UPV) se ubican en las primeras posiciones por número de patentes. Resultados similares son los obtenidos en el Ranking de investigación de las universidades públicas españolas (Buela-Casal y otros, 2010). En este ranking son 6 las universidades coincidentes (UPV, UPC, UMH, UNAVARRA, UAH y USC) en las 10 primeras posiciones con las del Observatorio IUNE. Siguiendo con el razonamiento anterior, habría que destacar el porcentaje de colaboración internacional que muestra la UPC, y que no es común observarlo en universidades politécnicas, ya que se sitúa entre las 10 primeras instituciones por este indicador, concretamente en la $5^{a}$ posición.

Los indicadores de competitividad muestran que varias de las universidades más activas en la obtención de proyectos de investigación por profesor, tanto en el ámbito nacional como en el europeo, son jóvenes y de pequeño tamaño, y dos de ellas, la UPF y la UC3M, son de las que muestran una mayor actividad en ambos ámbitos. En los resultados que Buela-Casal y otros, (2010) muestran para la obtención de proyectos nacionales, todas las instituciones que aparecen en las 5 primeras posiciones son universidades jóvenes y de pequeño tamaño, situándose la UPF y la UC3M en la $1^{a}$ y $4^{a}$ posición respectivamente. Estos resultados permitirían concluir que las universidades más recientes se han creado con unas estructuras más ágiles y competitivas que les permiten realizar una gestión más eficiente de sus recursos. También hay que señalar la competitividad que muestran en esta dimensión algunas de las universidades medianas o grandes como es el caso de la UAB y UB, que aparecen entre las 10 más activas del Observatorio IUNE y del Ranking de investigación de las universidades públicas españolas.

Los indicadores analizados permiten observar que existe un grupo de universidades que destacan en diferentes dimensiones. Tal como argumentan Corera y otros (2010) a propósito de los resultados obtenidos en su Ranking general y por áreas de las universidades españolas, las instituciones líderes son aquéllas que se posicionan en los primeros puestos de cada uno de los indicadores analizados y son, por lo tanto, las que pueden ser consideradas de excelencia. Sin embargo, esta excelencia es muy difícil de determinar, ya que la calidad está distribuida de manera desigual entre las instituciones españolas y la varianza es muy grande. Según los autores, esta situación pone a las universidades frente al desafío de la especialización ya que es imposible destacar en todos los campos.

Como ha podido observarse, las posiciones de las universidades en el Observatorio IUNE presentan coincidencias importantes en el núcleo de instituciones más activas con otros rankings, fundamentalmente con el Ranking de investigación de las universidades públicas españolas, sin embar- 
go, en aquellas instituciones que presentan valores más bajos en los indicadores se observa que sus posiciones son más erráticas en cada uno de los rankings analizados. Esto está vinculado con la metodología de trabajo, ya que cuando los valores de los indicadores de las universidades son muy elevados, los sesgos que se producen en los procesos metodológicos no modifican sustancialmente las posiciones de estas instituciones. Esto mismo ocurre en varios de los rankings internacionales (ARWU, THE) donde de manera general se puede decir que hay bastante coincidencia en las posiciones de las primeras instituciones, sin embargo, a medida que se avanza en la clasificación, las universidades se posicionan de una manera mucho menos consistente.

Algunas de las universidades públicas españolas, como la UAM, la UCM, la UB o la UV, aparecen como las instituciones españolas más destacadas de las 11 incluidas en el ranking ARWU de la edición 2011, mientras que la UCM, la UPC y la UPM son las primeras de las españolas si se considera el Ranking Web of World Universities. Las particularidades de cada ranking pueden hacer que destaquen otras universidades más pequeñas como la UPF o la UMH, en función de las variables utilizadas. Por ello, teniendo en cuenta la alta variación de la posición de las universidades y el impacto que esto puede tener en su prestigio, recomendamos el uso de los rankings a partir de múltiples indicadores, lo que puede resultar de suma utilidad a la hora de conocer aspectos concretos sobre una actividad determinada. Indudablemente es importante destacar también la utilidad de combinar este tipo de indicadores con las opiniones de expertos, ya que, las recomendaciones vertidas por miembros de la propia comunidad académica nacional e internacional pueden constituir un excelente complemento para comprender y valorar la actividad del sistema universitario.

Como conclusión queremos destacar que, aunque es imposible obviar la importancia y utilidad que han adquirido los rankings internacionales, de hecho son en gran medida los responsables de activar la competitividad entre las universidades a nivel mundial, lo que está repercutiendo en la mejora de sus resultados de investigación y docencia, sin embargo, no hay que perder de vista que en ellos reside una contradicción intrínseca. Como explica Muñoz García (2012), un ranking es una jerarquía que busca la distinción (identificar las "mejores" y las "peores" instituciones), sin embargo, crea un patrón único con el que medir, para que todas las universidades sigan una estrategia que las lleve a parecerse a las universidades que han alcanzado las primeras posiciones y esto enmascara la diversidad de un sistema. Asimismo, puede argumentarse que los rankings pueden presentar una imagen engañosa de la realidad, ya que se tiende a pensar que el lugar que ocupan las instituciones refleja de manera certera su calidad, $y$, por lo tanto, que el número de instituciones de un país en esos sitios de privilegio puede ser un indicador válido para evaluar la calidad de su sistema de educación superior (Martínez Rizo, 2012). En este sentido, hay que tener en cuenta que una universidad puede ser excelente en determinadas áreas, pero no en el conjunto. Esto hace que sea imprescindible interpretar adecuadamente los resultados que ofrecen los rankings.

Es por ello que consideramos que resulta esencial para las regiones y países no centrales generar alternativas a los rankings internacionales que permitan poner de manifiesto la realidad de cada uno de los sistemas universitarios. En ese sentido, valoramos positivamente las iniciativas surgidas en los últimos años en el contexto español, ya que están permitiendo contar con información detallada y valiosa sobre la actividad científica de las universidades. En esta misma línea creemos que el Observatorio IUNE puede contribuir de forma destacada para superar algunas de las limitaciones mencionadas, incluyendo en la próxima edición -prevista para el primer trimestre de 2013- el desglose de la actividad investigadora de las universidades por áreas temáticas.

\section{AGRADECIMIENTOS}

La creación del Observatorio IUNE ha sido posible gracias a la financiación recibida por el Ministerio de Educación, Cultura y Deporte a través de los proyectos "Desarrollo de un Observatorio de la actividad investigadora de las universidades españolas en el periodo 2002-2009, a partir de indicadores de I+D+i". (REF: EA2010-0042) y "Seguimiento y actualización de la actividad investigadora de las universidades españolas durante el período 2002-2010" (EA2011-0058). Asimismo, los autores quieren agradecer la colaboración de todos los miembros del equipo del proyecto, pertenecientes a las universidades Autónoma de Madrid, Autónoma de Barcelona, Pompeu Fabra y Carlos III de Madrid.

\section{NOTAS}

[1] Datos INE: Profesorado de los centros propios de las universidades por sexo y categoría (www.ine.es).

\section{BIBLIOGRAFÍA}

Aguillo, I; Bar-Illan, J; Levene, M; Ortega, J.L. (2010). Comparing university rankings. Scientometrics, vol 85 (1), 243-256.

Bordons, M; Sancho, R; Morillo, F; Gómez, I. (2010). Perfil de actividad científica de las universidades españolas en cuatro áreas temáticas: un enfoque multifactorial. Revista Española de Documentación Científica, 33 (1), 9-33, 2010.

Buela-Casal, G.; Bermúdez, M.P.; Sierra, J.C.; Quevedo-Blasco, R.; Castro, A. (2010). Ranking de 2009 en investigación de las universidades públicas españolas. Psicothema, 22, 171-179. 
Buela-Casal, G.; Bermúdez, M.P.; Sierra, J.C.; Quevedo-Blasco, R.; Castro, A.; Guillén-Riquelme, A. (2011). Ranking de 2010 en producción y productividad en investigación de las universidades públicas españolas. Psicothema, 23, 527-536.

Buesa, M; Hieijs, J; Kahwash, O. (2009). La calidad de las universidades en España. Elaboración de un índice multidimensional. Madrid: Consejo Económico y Social.

Clark, B. (1997). Las universidades modernas: espacios de investigación y docencia. México: Grupo Editorial de Miguel Ángel Porrúa/Coordinación de Humanidades/UNAM.

Corera, E; Chinchilla, Z; De-Moya, F; Sanz Menéndez, L. (2010). Producción científica e impacto: ranking general y por áreas de las instituciones universitarias españolas. En Informe CyD 2009. Barcelona: Fundación CyD, p. 254-262.

Delgado-López-Cózar, E; Jiménez-Contreras, E; RuízPérez, R. (2009). La ciencia española a través de la Web of Science (1996-2007): las disciplinas. El profesional de la Información, 18 (4), 437-443

Egron-Polak, E. (2007). Editorial, IAU Horizons, 13 (23), 1-2.

Gómez, I.; Sancho, R.; Bordons, M.; Fernández, M. T. (2006). La I+D en España a través de publicaciones y patentes. En: Sebastián, J. y Muñoz, E. (editores.). Radiografía de la Investigación pública en España. Madrid: Biblioteca Nueva, 273-301.

Gómez, I; Bordons, M; Fernández, M. T.; Morillo, F. (2009). Structure and research performance of Spanish universities. Scientometrics, 79 (1), 131-146.

International Ranking Expert Group. IREG. (2006). Berlin principles on Ranking of Higher Education Institutions. Disponible en: http://www.che.de/ downloads/Berlin_Principles_IREG_534.pdf. (Consulta: 10/02/2013).

Liu, N.C; Cheng, Y. (2005). Academic ranking of World Universities. Methodologies and Problems. Higher Education in Europe, 30 (2), 127-136.

Marginson, S.; Mollis, M. (2001). The door opens and the tiger leaps. Theories and reflexivities of comparative education for a global millennium. Comparative Education Review. 45(4), 581-615.

Marginson, S. (2012). Global university rankings: the strategic issues. En: Encuentro internacional Las universidades latinoamericanas ante los rankings internacionales. Impactos, alcances y límites. Ciudad de México, 17-18 de mayo. Disponible en: http://www.encuentro-rankings.unam.mx/Documentos/ConferenciaMagistralMarginsontexto.pdf. (Consulta: 10/02/2013).

Martínez Rizo, F (2012). Impactos deseables e indeseables en las Universidades de América Latina. En Encuentro internacional Las universidades latinoamericanas ante los rankings internacionales. Impactos, alcances y límites. Ciudad de México, 17-18 de mayo. Disponible en: http://www.encuentro-rankings.unam.mx/Documentos/ImpactoRankingsRizo.pdf. (Consulta: 10/02/2013).
Muñoz García, H. (2012). Comentarios a la ponencia de Simon Marginson. En Encuentro internacional Las universidades latinoamericanas ante los rankings internacionales. Impactos, alcances y límites. Ciudad de México, 17-18 de mayo. Disponible en: http://www.encuentro-rankings.unam.mx/Documentos/acto_munoz.pdf. (Consulta: 10/02/2013).

Orduña Malea, E. (2011). Propuesta de un modelo de análisis redinformétrico multinivel para el estudio sistémico de las universidades españolas (2010). [Tesis Doctoral]. Universidad Politécnica de Valencia. Disponible en: http://hdl.handle. net/10251/14420. (Consulta: 10/02/2013).

Sanz-Casado, E; García-Zorita, C; Iribarren-Maestro, I; García, P.E; Sánchez-Gil, S. (2008). Propuesta de evaluación de la investigación en la universidad española a partir de indicadores complementarios. En Bermúdez, Ma P. ; Ramiro, Ma T. (Comp). Evaluación de la calidad de la educación superior y de la investigación. V Foro de evaluación de la calidad universitaria, San Sebastián.

Torres-Salinas, D; Delgado-López-Cózar, E; GarcíaMoreno-Torres, J; Herrera, F (2011a). Ranking ISI de las universidades españolas según campos científicos: descripción y resultados. El profesional de la Información, 20 (1), 111-118.

Torres-Salinas, D.; Moreno-Torres, J.G.; Robinson, N.; Delgado López-Cózar, E.; Herrera, F. (2011b). Rankings ISI de las Universidades Españolas según Campos y Disciplinas Científicas [en línea]. 2.a ed. 2011. Disponible en: http://rankinguniversidades. es. (Consulta: 10/02/2013).

Torres-Salinas, D.; Cabezas-Clavijo, Á. (2012). Herramientas para la evaluación de la ciencia en universidades y centros I+D: descripción y usos. Anuario ThinkEPI, 6, 142-146.

van-Raan, A. (2005). Fatal attraction: conceptual and methodological problems in the ranking of universities by bibliometric methods. Scientometrics, 62 (1), 133-143.

van Vught, F; Kaiser, F; File, J; Gaethgens, C; Peter, R; Westerheijden, D. (2010). U-Map. The European classification of higher education institutions. [on line]. Center for Higher Education Policy Studies, University of Twente. Disponible en: http://www.umap.eu/. (Consulta: 10/02/2013).

van Vught, F.; Ziegele, F (2011). U-Multirank. The design and testing the feasibility of a multidimensional global university ranking. Final report. Consortium for Higher Education and Research Performance Assesment, CHERPA-Network. Disponible en: https:// www.ireg-observatory.org/pdf/u_multirank_final_ report.pdf. (Consulta: 10/02/2013).

Webster, D. (1983). America's Higher-ranked graduate Schools, 1925-1982. Change, May-June, pp.13-24.

Webster, D. (1985). How not to rank universities. Higher Education, 4 (1), 71-79.

Webster, D. (1986). Academic Quality Rankings of American Colleges and Universities. Springfield, IL. : Charles C. Thomas Publisher. 
ANEXO I: listado de universidades y acrónimos

\begin{tabular}{|c|c|}
\hline Universidad & COD Observatorio \\
\hline Universidad de Alicante & UA \\
\hline Universidad de Extremadura & UNEX \\
\hline Universidad de las Illes Balears & UIB \\
\hline Universidad de Barcelona & UB \\
\hline Universidad de Cádiz & UCA \\
\hline Universidad de Córdoba & UCO \\
\hline Universidad de Santiago de Compostela & USC \\
\hline Universidad de Granada & UGR \\
\hline Universidad de León & UNILEON \\
\hline Universidad Complutense de Madrid & UCM \\
\hline Universidad de Málaga & UMA \\
\hline Universidad de Murcia & UM \\
\hline Universidad de Oviedo & UNIOVI \\
\hline Universidad de Salamanca & USAL \\
\hline Universidad de La Laguna & ULL \\
\hline Universidad de Cantabria & UNICAN \\
\hline Universidad de Sevilla & US \\
\hline Universitat de València (Estudi General) & UV \\
\hline Universidad de Valladolid & UVA \\
\hline Universidad del País Vasco/Euskal Herriko Unibertsitatea & EHU \\
\hline Universidad de Zaragoza & UNIZAR \\
\hline Universidad Autónoma de Barcelona & UAB \\
\hline Universidad Autónoma de Madrid & UAM \\
\hline Universidad Politécnica de Catalunya & UPC \\
\hline Universidad Politécnica de Madrid & UPM \\
\hline Universidad de Las Palmas de Gran Canaria & ULPGC \\
\hline Universidad Politécnica de Valencia & UPV \\
\hline Universidad Nacional de Educación a Distancia & UNED \\
\hline Universidad de Alcalá & UAH \\
\hline Universidad de Castilla-La Mancha & UCLM \\
\hline Universidad Pública de Navarra & UNAVARRA \\
\hline Universidad Carlos III de Madrid & UC3M \\
\hline Universidad de A Coruña & UDC \\
\hline Universidad de Vigo & UVIGO \\
\hline Universidad Pompeu Fabra & UPF \\
\hline Universidad Jaume I de Castellón & UJI \\
\hline Universidad Rovira i Virgili & URV \\
\hline Universidad de Girona & UDG \\
\hline Universidad de Lleida & UDL \\
\hline Universidad de la Rioja & UNIRIOJA \\
\hline Universidad de Almería & UAL \\
\hline Universidad de Huelva & UHU \\
\hline Universidad de Jaén & UJAEN \\
\hline Universidad de Burgos & UBU \\
\hline Universidad Miguel Hernández de Elche & UMH \\
\hline Universidad Rey Juan Carlos & URJC \\
\hline IE Universidad & SEK \\
\hline Universidad Pablo de Olavide & UPO \\
\hline Universidad Politécnica de Cartagena & UPCT \\
\hline
\end{tabular}

